\title{
A giant pedunculated uterine leiomyoma with cystic and mucinous degeneration presenting as an ovarian malignancy
}

Selçuk Gülmez ${ }^{1}$, Nesrin Baştuğ ${ }^{2}$, Aziz Serkan Senger ${ }^{1}$, Ulaş Aday ${ }^{1}$, Orhan Uzun ${ }^{1}$, Emre Bozdağ ${ }^{1}$, Durmuş Ali Çetin ${ }^{1}$

${ }^{1}$ Gastroenterological Surgery Clinic, University of Health Sciences, Kartal Kosuyolu High Specialty Training and Research Hospital, Istanbul, Turkey

${ }^{2}$ Obstetric and Gynecology Department, Emsey Hospital, Istanbul, Turkey

Submitted: 10 June 2017

Accepted: 21 July 2017

Arch Med Sci Civil Dis 2017; 2: e145-e147

DOI: https://doi.org/10.5114/amscd.2017.71207

Copyright $\odot 2017$ Termedia \& Banach

Leiomyomas of the uterus (myomas or fibroids) are benign tumours originating from the smooth muscle cells of the myometrium, and their prevalence parallels that of changes in levels of oestrogen and progesterone, which are reproductive hormones. While their incidence rate increases during reproductive age, it decreases after menopause. The image of a homogeneous or heterogeneous hypoechoic uterine mass as revealed by ultrasonography (USG) expedites diagnosis, but imaging methods may prove to be insufficient in differentiating the organ that the mass originates from if there is an increase in size together with degeneration. In such cases, leiomyomas of the uterus, especially pedunculated ones, may not be successfully differentiated from malign masses of the uterus [1, 2]. We present a case of a giant degenerated leiomyoma presenting as an ovarian malignancy.

A 39-year-old female patient presented with complaints of progressive abdominal fullness, sporadic nausea and abdominal pain. The anamnesis of the patient revealed that she had had two vaginal births, while her physical examination and routine laboratory tests including tumour markers were normal. The patient's abdominal USG showed a giant, semisolid mass filling up the pelvis and the abdomen. Her abdominal magnetic resonance imaging (MRI) revealed a giant mass which was thought to be originating from the left adnexal area, which displaced the uterus towards the right. Differentiation of the bladder and uterus border could not be made. The mass contained solid-cystic components and demonstrated intensive contrast involvement (Figure $1 \mathrm{~A}$ ). There were also widespread, conglomerate, para-aortic lymph nodes of about $17 \mathrm{~mm}$ in size. The MRI diagnosis was reported to be suspected cystadenocarcinoma of the left ovary.

Median laparotomy was performed on the patient. The exploration showed a multilobular giant mass filling up the whole abdomen, originating from the fundal posterior wall with soft consistency and smooth borders, containing pseudocysts on the periphery (Figures $1 \mathrm{~B}, \mathrm{C}$ ). At the request of the patient the uterus was preserved and the mass was totally extirpated as she was fertile. The uterine defect was primarily repaired. The mass was $35 \times 30 \times 10 \mathrm{~cm}$ in size and weighed $5.5 \mathrm{~kg}$ (Figure $1 \mathrm{C}$ ). The results of the pathological analysis revealed fusiform shaped smooth muscle bundles with a uniform nucleus that formed vortex-like structures.
Corresponding author:

Selçuk Gülmez

Gastroenterological

Surgery Clinic

University of

Health Sciences

Kartal Kosuyolu

High Specialty

Training and

Research Hospital

34865 Istanbul, Turkey

Phone: +90 5530846215

E-mail: selcukgulmez54@

hotmail.com 

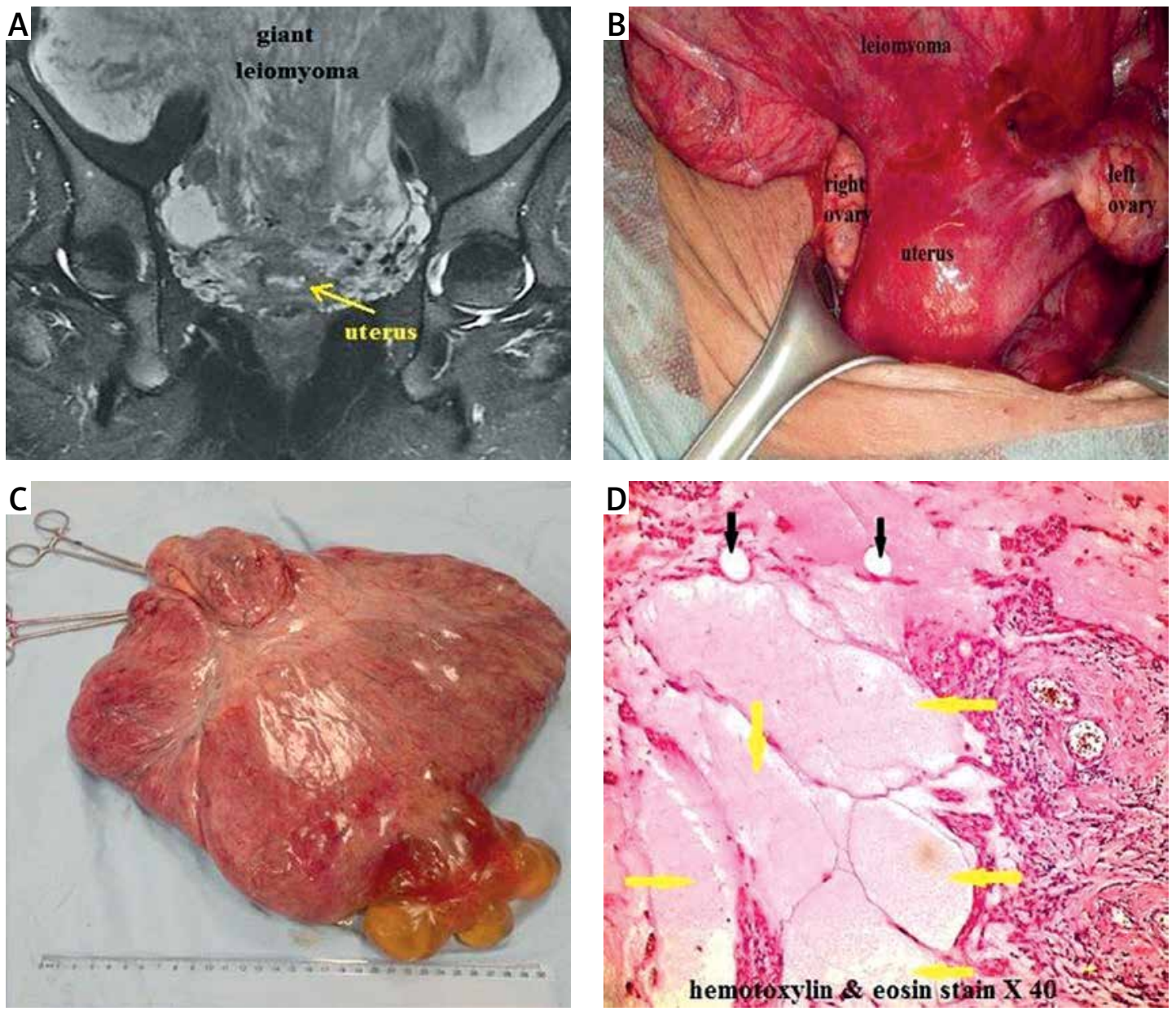

Figure 1. A - Coronal T2-weighted magnetic resonance imaging of the giant leiomyoma. B - Uterine leiomyoma with peduncle. $\mathbf{C}$ - The giant mass after resection $(35 \times 30 \times 10 \mathrm{~cm}$ and $5.5 \mathrm{~kg})$. D - Mucinous (yellow arrows) and cystic degeneration (black arrows)

No cellular atypia or necrosis was seen. There were isolated mitotic figures. The final pathological diagnosis was reported to be leiomyoma with cystic and mucinous degenerations of the uterus (Figure $1 \mathrm{D}$ ). The patient was discharged on the $3^{\text {rd }}$ post-operative day from our clinic.

Leiomyomas are the most common uterine neoplasm and they occur in $25 \%$ of women of reproductive age [3]. According to their location, leiomyomas are classified as submucosal, intramural or subserosal. Subserosal leiomyomas may be large and pedunculated and simulate ovarian neoplasms [4].

As leiomyomas enlarge, they may outgrow their blood supply, resulting in various types of degeneration. These include hyaline, cystic, myxoid or red degeneration and dystrophic calcification. Hyalinisation is the most common type of degeneration, occurring in up to $60 \%$ of cases. Cystic degeneration, observed in about $4 \%$ of leiomyomas, may be considered an extreme sequela of oedema [2].

Myomas may be formed as singular or multiple tumours that are millimetric or as large as $10 \mathrm{~cm}$ in size [5]. Although a great majority of myomas are small and asymptomatic, many women experience serious problems at a certain stage in their lives and need treatment. The symptoms are related to the number, size, and localization of the myomas. Myoma-related symptoms are generally classified under three categories: (1) increase in uterine haemorrhage, (2) pelvic pressure and pain, symptoms related to the effects of the mass, and (3) reproductive dysfunction [6].

Transvaginal USG is the most frequently used diagnostic and imaging method, because of its accessibility and cost-effectiveness [7]. Magnetic resonance imaging, on the other hand, is the best modality in showing the sizes and locations of all uterine myomas as well as differentiating leiomyoma, adenomyoma, and adenomyosis. Performing MRI before uterine artery embolization may prove to be very useful as it may enable the differentiation of leiomyomas from leiomyosarcomas and the prediction of uterine artery embolization results beforehand [8, 9]. Computed tomography (CT) is not the primary modality for diagnosing or evaluating leiomyomas. Solid mass type or dense and amorphous calcifications in a uterine mass 
are the most specific sign for a leiomyoma; however, these occur in only $10 \%$ of cases [2].

Treatment options of uterine leiomyomas include surgery, medical or hormonal treatment, myolysis, and uterine artery embolization. Most myomas do not require intervention unless they cause symptoms. For symptomatic myomas, hysterectomy offers a definitive solution. The treatment should be individualized according to many factors including the patient's age, fertility status, the severity and type of symptoms, suspicion of malignancy, the location and size of myomas, and desire of the patient. For women of reproductive age who would like to maintain their fertility or those who want to preserve their uterus myomectomy remains the treatment of choice and the gold standard $[5,6]$.

Giant leiomyoma with cystic degeneration is a rare condition. It may sometimes present with clinical and imaging findings giving the impression of another diagnosis such as an ovarian mass, the final diagnosis depending on laparotomy findings with histological confirmation [1]. In our case, neither USG nor MRI was able to verify the ovaries as different organs from the uterus because of the giant mass which was adjacent to both ovaries, filling up the pelvis completely. This verification failure caused our pre-diagnosis to be focused on the mass originating from the ovary, predicting an ovarian tumour. Additionally, the mass's large size and multilocularity are characteristics of a cystadenoma or cystadenocarcinoma. But the final histological diagnosis of the mass was uterine leiomyoma with cystic degeneration. This case showed that degenerated myomas, especially pedicled ones, can easily be confused with ovarian tumours and should be taken into consideration in the differential diagnosis.

\section{Conflict of interest}

The authors declare no conflict of interest.

\section{References}

1. Sambath S, Balasubramanian T. A giant pedunculated leiomyoma with cystic degeneration mimicking an ovarian malignancy. Int J Reprod Contracept Obstet Gynecol 2016; 5: 252-4.

2. Low SCA, Chong CL. A case of cystic leiomyoma mimicking an ovarian malignancy. Ann Acad Med Singapore 2004; 33: 371-4.

3. Naykı Ü, Naykı C, Uluğ P, Yılmaz İ, Cetin Z, Yıldırım Y. A rare case of a giant cystic leiomyoma presenting as a retroperitoneal mass. Iran J Reprod Med 2014; 12: 831-4.

4. Aydin C, Eris S, Yakin Y, Yalcin Y, Selim HS. A giant cystic fibroid mimicking ovarian malignancy. Int I Surg Case Rep 2013; 4: 1010-2.

5. Aksoy H, Aydin T, Özdamar Ö, Idem Karadag Öi, Aksoy U. Successful use of laparoscopic myomectomy to remove a giant uterine myoma: a case report. J Med Case Rep 2015; 9: 286.

6. Karaman E, Cim N, Bulut G, et al. A case of giant uterine lipoleiomyoma simulating malignancy. Case Rep Obstet Gynecol 2015; 2015: 926961.

7. Sharma RP, Sharma N, Sharma K, et al. Int J Med Sci Clin Invention 2015; 2: 640-4.

8. Omary, RA, Vasireddy, S, Chrisman, HB, et al. The effect of pelvic MR imaging on the diagnosis and treatment of women with presumed symptomatic uterine fibroids. J Vasc Interv Radiol 2002; 13: 1149-53.

9. Vedantham S, Sterling KM, Goodwin SC, et al. I. Uterine fibroid embolization: preprocedure assessment. Tech Vasc Interv Radiol 2002; 5: 2-16. 